\title{
Hot gas in Milky Way size galaxies at $\mathrm{z}=0$
}

\author{
Santi Roca-Fàbrega ${ }^{1}$, Pedro Colin ${ }^{2}$, Octavio Valenzuela ${ }^{3}$, Francesca $^{2}$ \\ Figueras $^{4}$ and Yair Krongold ${ }^{3}$ \\ ${ }^{1}$ Racah Institute of Physics, The Hebrew University, Jerusalem 91904, Israel \\ email: santi.roca@mail.huji.ac.il \\ ${ }^{2}$ Instituto de Radioastronomía y Astrofísica, UNAM, Morelia, Michoacán 58089, México. \\ ${ }^{3}$ Instituto de Astronomía, UNAM, Ciudad Universitaria, México D.F. \\ ${ }^{4}$ IEEC-ICCUB, Martí i Franquès, 1, E-08028 Barcelona.
}

\begin{abstract}
We present a new set of cosmological Milky Way size galaxy simulations using ART. In our simulations the main system has been evolved inside a $28 \mathrm{Mpc}$ cosmological box with a spatial resolution of $109 \mathrm{pc}$. At $\mathrm{z}=0$ our systems have an $\mathrm{M}_{v i r}=6-8 \times 10^{11} \mathrm{M}_{\odot}$. In several of out models we have observed how a well defined disk is formed inside the dark matter halo and the overall amount of gas and stars is comparable with MW observations. Several non-axisymmetric structures arise out of the disk: spirals, bars and also a warp. We have also observed that a huge reservoir of hot gas is present at large distances from the disk, embedded in the dark matter halo region, accounting for only a fraction of the "missing baryons". Gas column density, emission (EM) and dispersion (DM) measure have been computed from inside the simulated disk at a position of $8 \mathrm{kpc}$ from the center and in several directions. Our preliminary results reveal that the distribution of hot gas is non-isotropic according with observations (Gupta et al. 2012, Gupta et al. 2014). Also its metallic content presents a clear bimodality what is a consequence of a recent accretion of a satellite galaxy among others. After a careful analysis we confirm that due to the anisotropy in the gas distribution a new observational parameter needs to be defined to recover the real distribution of hot gas in the galactic halo (Roca-Fàbrega et al. 2016).
\end{abstract}

Keywords. galaxies: formation - methods: numerical - Galaxy: halo - galaxies: intergalactic medium

\section{Introduction}

Nowadays it is commonly accepted that the amount of baryons observed in the local universe is much below the one inferred from cosmology. This lack of baryons in galaxies is known as the "missing baryons problem".

To solve this missing baryons problem several authors proposed that galactic winds, SNe feedback or strong AGN winds ejected part of the galactic baryons to the dark matter halo and circumgalactic medium (CGM) as hot gas. Others authors proposed that also part of the gas never collapsed into the dark matter halos as it was previously heated by SNe of Population III.

From the point of view of large volume hydrodynamical galaxy formation simulations, some attempts have been made in order to investigate the presence and detectability of hot gas halo corona (e.g. Mollitor et al. 2014) and some succeeded in obtaining realistic results when compared with observations (e.g. Crain et al. 2013).

From an observational point of view, first detections where done by Forman et al.(1985) in external galaxies and later on several authors get better detections in other systems and studied them deeper (e.g. Bogdan \& Gilfanov 2011)). These detections were done by the analysis of X-ray absorption lines from OVII and OVIII which only exist in environments with temperatures between $10^{6}$ and $10^{7} \mathrm{~K}$. The problem of using this technique is that these X-ray absorption lines only can be observed in the directions of extragalactic 
luminous sources (QSO, AGN, ...) or galactic X-ray emitters as X-ray binaries.

Here we have studied how the hot gas component embedded in the DM halo can account for part of the missing baryons in galaxies and also its distribution and properties, using new high resolution simulations. We also studied possible observational biases in the determination of total hot gas mass, when only a small number of line of sight observations are used.

\section{GARROTXA models}

Simulations used here have been run using the Eulerian hydrodynamics + N-body ART code (Kravtsov et al. 1997, Kravtsov et al. 2003) and the commonly used zoom-in technique. Selected DM halos have had not major mergers since $\mathrm{z}=1.5$ and that at $\mathrm{z}=0$ have not a a similar mass companion inside a sphere of $1 \mathrm{Mpc} \mathrm{h}^{-1}$. For more details on the code and halo selection process see Colin et al. (2010) and Roca-Fàbrega et al. (2016).

All selected halos have been simulated up to $\mathrm{z}=0$. At this time all of them host a massive disk galaxy with several non-axisymmetric structures such as bars or spirals. The disk scale height and length in our models depends on the age of the stellar population, while the former is higher the latter is smaller when the population gets older. Gas distribution in all our models is not isotropic and depends on gas temperature: cold gas is present inside the young stellar disk and hot gas fills the out-of-plane region and is embedded in the DM halo.

\section{Discussion and conclusions}

\subsection{Hot gas spatial distribution and origin}

We define hot gas as gas with $\mathrm{T}>3 \times 10^{5} \mathrm{~K}$. This definition is observationally motivated as real hot gas mass is inferred from observations of the ionized oxygen emission (Gupta et al. 2012). In this work we computed the hot gas column density, emission measure and dispersion measure from a position that is at $8 \mathrm{kpc}$ from the galactic center, inside the simulated galactic disk, resembling the Sun position, and assuming arbitrary azimuthal angles. We have obtained values that fall near the observational ranges, if a solar metallicity is assumed. The distribution of hot gas inferred from all estimations is far from homogeneous (see Fig.1). In order to quantify the hot gas anisotropy we computed the amplitude of the first spherical harmonics ( $\mathrm{Y}_{l}^{m}$ from $\mathrm{l}, \mathrm{m}=0$ to 5 ) and the filling factor. Our results show that the dominant spherical harmonic is $Y_{1}^{0}$, that is an indicator of the dipolar component. The computation of the mean filling factor from line of sights distributed all along the sky gives as a value of $\mathrm{f}_{v} \sim 0.33 \pm 0.15$. This value for the filling factor also suggests that hot gas is concentrated in a few regions of the sky.

We also analyzed the metals distribution and we have found that it exists a clear bimodality between the northern and southern latitudes and also that several vertical motions and metallicity gradients are present in the hot gas halo. We have found that some low metallic gas is falling from the IGM (cold flows) and hot metallic departs from the stellar disk region (SN feedback, stellar winds...). We have also observed that some hot metallic gas is also falling to the system, associated with a small gaseous satellite that is passing through the system at $\mathrm{z}=0$.

\subsection{Missing baryons, halo virial mass and the total hot gas in galaxies}

The baryonic fraction in our simulated galaxies reaches $(0.107-0.120)$. These values are still far from the cosmic baryon fraction $\left(\mathrm{F}_{b, U}=0.17\right)$ even after adding the hot gas mass 


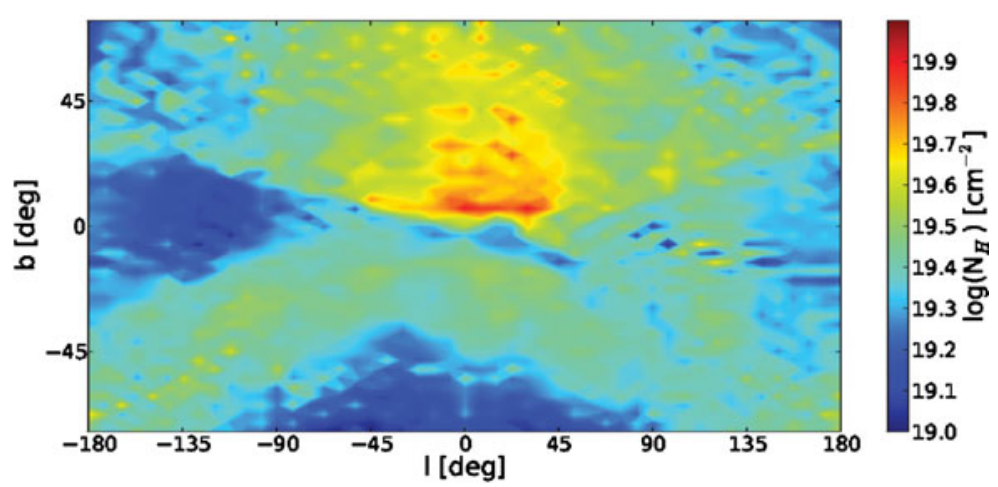

Figure 1. Hot gas column density (top) in a full sky view in galactic coordinates, at $\mathrm{z}=0$.

component inside halos. However, because we do not include the feedback effect of an AGN, that may eject the gas outside the halo, we can consider our estimates as an upper limit to the halo hot gas mass. We argue that the still missing baryons must be placed both in the IGM and along the filaments and far from the main galactic systems.

Other result we have obtained after analyzing several models of MW-sized systems, a few of more massive or lighter and also some with different feedback recipes, is that $\mathrm{M}_{v i r}$ correlates well with total hot gas mass. If finally confirmed this linear correlation will provide a new constrain to virial mass in galaxies, including the Milky Way.

\subsection{Observational biases}

We have used simulations to detect possible observational biases that arise from observational technique and used assumptions. With such aim we have located a mock observer at $8 \mathrm{kpc}$ from the galactic center inside our MW-sized simulations. We have computed the column density and the Emission Measure, and from them the total galactic hot gas mass. We have found that using the spherical uniform approach, the total hot gas mass is systematically overestimated or underestimated depending on the technique (see RocaFàbrega et al. 2016). This result is a consequence of that the real density distribution of our model is not uniform but decreasing with radius as a power law.

As a conclusion we state that using both, column density plus imposed optical depth or a combination of column density and emission measure, we are not able to obtain the real hot halo gas mass, when assuming it is isotropically distributed in the galactic halo. Nevertheless, these measurements, particularly the observational method in the literature using both, $\mathrm{N}_{H}$ and EM are useful to get an order of magnitude estimate of the total hot gas mass of the halo. The work is in progress to find the density profile that will allow us to get the best hot halo gas mass estimation, from observations.

\section{References}

Bogdan, A. \& Gilfanov, M. 2011, MNRAS, 418, 1901

Colin, P., Avila-Reese, V., Vazquez-Semadeni, E., et al. 2010, ApJ, 713, 535

Crain, R. A., McCarthy, I. G., Schaye, J., et al. 2013, MNRAS, 432, 3005

Gupta, A., Mathur, S., Krongold, Y., Nicastro, F., \& Galeazzi, M. 2012, ApJ, 756, L8

Gupta, A., Mathur, S., Galeazzi, M., \& Krongold, Y. 2014, Ap\&SS, 352, 775

Kravtsov, A. V., Klypin, A. A., \& Khokhlov, A. M. 1997, ApJS, 111, 73

Kravtsov A. V. 2003, ApJ, 590, L1

Mollitor P., Nezri E., Teyssier R. 2015, MNRAS, 447, 1353

Roca-Fàbrega S., Valenzuela O., Colin P., et al. 2016, ApJ, submitted 